Pacific Journal of Mathematics

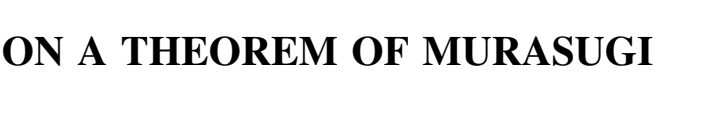




\section{ON A THEOREM OF MURASUGI}

\section{McA. Gordon ANd R. A. Litherland}

1. Let $l=k_{1} \cup k_{2}$ be a 2-component link in $S^{3}$, with $k_{2}$ unknotted. The 2 -fold cover of $S^{3}$ branched over $k_{2}$ is again $S^{3}$; let $k_{1}^{(2)}$ be the inverse image of $k_{1}$, and suppose that $k_{1}^{(2)}$ is connected. How are the signatures $\sigma\left(k_{1}\right), \sigma\left(k_{1}^{(2)}\right)$ of the knots $k_{1}$ and $k_{1}^{(2)}$ related? This question was considered (from a slightly different point of view) by Murasugi, who gave the following answer [Topology, 9 (1970), 283-298].

Theorem 1 (Murasugi).

$$
\sigma\left(k_{1}^{(2)}\right)=\sigma\left(k_{1}\right)+\xi(l) .
$$

Recall [4] that the invariant $\xi(l)$ is defined by first orienting $l$, giving, an oriented link $\bar{l}$, say, and then setting $\lessgtr(l)=$ $\sigma(\bar{l})+L k\left(\bar{k}_{1}, \bar{k}_{2}\right)$, where $\sigma$ denotes signature and $L k$ linking number.

In the present note we shall give an alternative, more conceptual, proof of Theorem 1 , and in fact obtain it as a special case of a considerably more general result.

The idea of our proof is the following. If $l=l_{1} \cup l_{2}$ is a link, partitioned into two sublinks $l_{1}$ and $l_{2}$, then the 2-fold branched covers over $l_{1}, l_{2}$, and the whole of $l$, are all quotients of a $\boldsymbol{Z}_{2} \oplus \boldsymbol{Z}_{2}$ cover branched over $l$. After possibly multiplying by 2 , the diagram consisting of these branched covers bounds a corresponding diagram of 4-manifolds, and the signatures of the various links involved are expressible in terms of the signatures of these 4-manifolds (and the euler numbers of the branch sets); see e.g., [3]. The result is then a consequence of a relation among these 4-manifold signatures (Lemma 1).

This more general setting requires that we consider links in 3 -manifolds other than homology spheres; in $\S 2$ we discuss the signature in this context. (It becomes necessary to prescribe a particular 2-fold branched cover. However, we sacrifice some generality inasmuch as we restrict ourselves to oriented, nullhomologous links: it would otherwise be necessary to prescribe a framing of the link as well.) In $\S 3$ we set up the diagram of covering spaces, and in $\S 4$ derive the relation between the signatures of the manifolds therein. Section 5 contains some consequences of this, including the appropriate generalization of Theorem 1.

All manifolds of dimensions 3 and 4 are to be oriented; manifolds of dimensions 1 and 2 are oriented only when this is explicitly 
stated, and those of dimension 2 need not even be orientable. We make no assumptions on the connectedness of our manifolds. If $\bar{l}$ is an oriented link, we denote the underlying nonoriented link by $l$.

2. Let $\bar{l}=\bar{k}_{1} \cup \cdots \cup \bar{k}_{m}$ be an oriented link in a closed 3-manifold $M$, and suppose $\bar{l}$ is null-homologous. Let $W$ be a 4-manifold and $F$ a surface in $W$ such that $\partial(W, F)=(M, l)$. Let $F^{\prime}$ be (the image of) a section of the normal $S^{1}$-bundle of $F$ in $W$, with $\partial F^{\prime}=l^{\prime}=k_{1}^{\prime} \cup \cdots \cup k_{m}^{\prime}$, say. Orient $l^{\prime}$ to obtain $\bar{l}^{\prime}=\bar{k}_{1}^{\prime} \cup \cdots \cup \bar{k}_{m}^{\prime}$ by requiring $\bar{k}_{i}^{\prime} \sim \bar{k}_{i}$ in a tubular neighborhood of $k_{i}$, and define $\bar{e}(F)=-L k\left(\bar{l}, \bar{l}^{\prime}\right)$. (Note that this is well-defined as $\bar{l}, \bar{l}^{\prime}$ are both null-homologous in $M$.)

Now let $p: \widetilde{M} \rightarrow M$ be some 2-fold covering of $M$ branched along $l$, and suppose that $p$ extends to a 2 -fold covering $\widetilde{W} \rightarrow W$ branched along $F$. Then

$$
\sigma(\bar{l}, p)=\sigma(\widetilde{W})-2 \sigma(W)+\frac{1}{2} \bar{e}(F)
$$

depends only on $\bar{l}$ and $p$. (If $\left(W_{1}, F_{1}\right)$ and $\left(W_{2}, F_{2}\right)$ are two pairs as above, apply the $G$-signature theorem [1] to the resulting involution on the closed 4-manifold $\widetilde{W}_{1} \bigcup_{\partial}-\tilde{W}_{2}$, together with Novikov additivity and the fact that the euler number of the normal bundle of $F_{1} \cup F_{2}$ in $W_{1} \cup-W_{2}$ is equal to $\bar{e}\left(F_{1}\right)-\bar{e}\left(F_{2}\right)$.)

We remark that if $M$ is a homology sphere, $p$ is unique, and $\sigma(\bar{l}, p)$ is just the signature of $\bar{l}$. Again, we may take $l$ to be the empty link; $-\sigma(\phi, p)$ is the $\alpha$-invariant [2] of the nontrivial covering translation of $\widetilde{M}$.

3. Let $l_{1}, l_{2}$ be disjoint links in a 3-manifold $M$, and write $l=l_{1} \cup l_{2}$. Let $\alpha: H_{1}(M-l) \rightarrow Z_{2} \oplus Z_{2}$ be a homomorphism which sends each meridian of $l_{1}$ (resp. $l_{2}$ ) to the nontrivial element of the first (resp. second) $Z_{2}$. Let $W$ be a 4 -manifold and $F_{1}, F_{2}$ disjoint surfaces in $W$ such that $\partial\left(W, F_{1}, F_{2}\right)=\left(M, l_{1}, l_{2}\right)$. Write $F=F_{1} \cup F_{2}$, and suppose there exists a homomorphism $\beta: H_{1}(W-F) \rightarrow \boldsymbol{Z}_{2} \oplus \boldsymbol{Z}_{2}$ such that $\alpha=\beta i_{*}$, where $i: M-l \rightarrow W-F$ is inclusion. (We shall discuss this assumption later.)

Let $\widetilde{W} \rightarrow W$ be the branched covering associated with $\beta$. The covering translations induce a $\boldsymbol{Z}_{2} \oplus \boldsymbol{Z}_{2}$-action on $\widetilde{W}$. Let $g_{1}$ generate the second $Z_{2}$ factor, $g_{2}$ the first, and let $g_{3}=g_{1} g_{2}$ be the remaining nontrivial element. Setting $W^{(i)}=\tilde{W} /\left(g_{i}\right), i=1,2,3$, we have the following commutative diagram of 2 -fold branched coverings. 


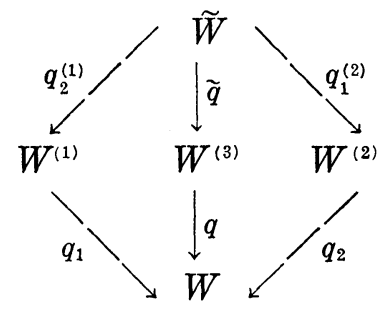

Here $q_{i}$ is branched over $F_{i}, i=1,2$, and $q$ is branched over $F$. If $F_{1}^{(2)}$ and $F_{2}^{(1)}$ are the inverse images of $F_{1}$ in $W^{(2)}$ and $F_{2}$ in $W^{(1)}$, then $q_{1}^{(2)}, q_{2}^{(1)}$ are branched over $F_{1}^{(2)}, F_{2}^{(1)}$ respectively. Finally, $\widetilde{q}$ is unbranched.

Now suppose that $l_{1}$ and $l_{2}$ can be oriented to obtain nullhomologous links $\bar{l}_{1}$ and $\bar{l}_{2}$ respectively. Let $\bar{l}=\bar{l}_{1} \cup \bar{l}_{2}$. There are induced orientations of $l_{1}^{(2)}=\partial F_{1}^{(2)}$ and $l_{2}^{(1)}=\partial F_{2}^{(1)}$, giving null-homologous links $\bar{l}_{1}^{(2)}$ and $\bar{l}_{2}^{(1)}$ in $\partial W^{(2)}$ and $\partial W^{(1)}$ respectively.

Writing $p$ 's instead of $q$ 's to denote the restrictions of these coverings to the appropriate boundaries, we have the equations

$$
\begin{gathered}
\sigma\left(\bar{l}_{1}, p_{1}\right)=\sigma\left(W^{(1)}\right)-2 \sigma(W)+\frac{1}{2} \bar{e}\left(F_{1}\right) \\
\sigma\left(\bar{l}_{2}, p_{2}\right)=\sigma\left(W^{(2)}\right)-2 \sigma(W)+\frac{1}{2} \bar{e}\left(F_{2}\right) \\
\sigma(\bar{l}, p)=\sigma\left(W^{(3)}\right)-2 \sigma(W)+\frac{1}{2} \bar{e}(F) \\
\sigma\left(\bar{l}_{1}^{(2)}, p_{1}^{(2)}\right)=\sigma(\widetilde{W})-2 \sigma\left(W^{(2)}\right)+\frac{1}{2} \bar{e}\left(F_{1}^{(2)}\right) \\
\sigma\left(\bar{l}_{2}^{(1)}, p_{2}^{(1)}\right)=\sigma(\widetilde{W})-2 \sigma\left(W^{(1)}\right)+\frac{1}{2} \bar{e}\left(F_{2}^{(1)}\right) \\
\sigma(\phi, \widetilde{p})=\sigma(\widetilde{W})-2 \sigma\left(W^{(3)}\right) .
\end{gathered}
$$

We now consider the question of the existence of a suitable homomorphism $\beta$. Suppose $H_{1}\left(W ; Z_{2}\right)=0$. Then (see $[3, \S 1]$ ) the cohomology exact sequence of the pair $(W, W-F)$, together with duality, gives an exact sequence

$$
\begin{aligned}
0 \longrightarrow H^{1}\left(W-F ; \boldsymbol{Z}_{2}\right) \longrightarrow & H_{2}\left(\boldsymbol{F}, \partial \boldsymbol{F} ; \boldsymbol{Z}_{2}\right) \longrightarrow H_{2}\left(W, \partial W ; \boldsymbol{Z}_{2}\right) \\
& H^{0}\left(\boldsymbol{F} ; \boldsymbol{Z}_{2}\right) .
\end{aligned}
$$

The existence of $\beta: H_{1}(W-F) \rightarrow Z_{2} \oplus Z_{2}$ taking a meridian of $F_{i}$ to the nontrivial element of the $i$ th $Z_{2}, i=1,2$, is then seen to be equivalent to the condition that $\left[F_{i}, \partial F_{i}\right]=0 \in H_{2}\left(W, \partial W ; Z_{2}\right)$, for $i=1$, 2. (In particular, the assertion $H^{1}\left(W-F ; Z_{2}\right) \cong H^{\circ}\left(F ; Z_{2}\right)$ in $[3$, p. 353] is incorrect.) 
Now suppose, in addition, that $H_{1}\left(M ; Z_{2}\right)=0$. Then $\beta$ will automatically satisfy $\alpha=\beta i_{*}$. But $\beta$ will not in general exist, for it is clear that if $\left[F_{i}, \partial F_{i}\right]=0 \in H_{2}\left(W, \partial W ; Z_{2}\right), i=1,2$, then $L k_{Z_{2}}\left(l_{1}, l_{2}\right)=0$. However, this condition is also sufficient; that is, given links $l_{1}, l_{2} \subset M$ such that $H_{1}\left(M ; Z_{2}\right)=0$ and $L k_{Z_{2}}\left(l_{1}, l_{2}\right)=0$, there exist $W, F_{1}, F_{2}, \beta$ as above. To see this, let $W$ be any 4 -manifold with $\partial W=M$ and $H_{1}\left(W ; \boldsymbol{Z}_{2}\right)=0$, and let $E_{1}, E_{2}$ be connected surfaces in $W$ with $\partial E_{i}=l_{i}$ and $\left[E_{i}, \partial E_{\imath}\right]=0 \in H_{2}\left(W, \partial W ; Z_{2}\right), i=1,2$. (For example, we could obtain $E_{i}$ by starting with a connected surface in $M$ bounded by $l_{i}$ and pushing its interior slightly into $W$.) We may assume that $E_{1}$ and $E_{2}$ intersect transversally in points in int $W$. Since $L k_{Z_{2}}\left(l_{1}, l_{2}\right)=0$, there will be an even number of such intersection points, and these may be removed, a pair at a time, by adding a tube to (say) $E_{1}$ along an arc in $E_{2}$ connecting the two points in question.

REMARK. Section 5 contains equations, derived from (i)-(vi) above, involving link signatures and linking numbers. Since both are additive under disjoint union, these equations will still be valid if we only assume $\partial\left(W, F_{1}, F_{2}\right)=k\left(M, l_{1}, l_{2}\right)$, the disjoint union of $k$ copies of $\left(M, l_{1}, l_{2}\right)$, for some $k>0$. Moreover, we have just seen that this weaker assumption is always satisfied (with $k=2$ ) if $M$ is a $\boldsymbol{Z}_{2}$-homology sphere. For notational simplicity, however, we shall continue to take $k=1$, without further comment.

4. To deduce relations between the link signatures on the left of equations (i)-(vi), we must find relations between the quantities on the right. The main ingredient is the following.

LEMMA 1.

$$
\sigma(\widetilde{W})=\sum_{i=1}^{3} \sigma\left(W^{(i)}\right)-2 \sigma(W)
$$

Proof. If $G$ is a finite group and $N$ is a $G$-manifold, then a standard transfer argument shows that

$$
\sigma(N)=|G| \sigma(N / G)-\sum_{g \in G-\{1\}} \operatorname{sign}(g, N) .
$$

Applying this to the $Z_{2} \oplus \mathbb{Z}_{2}$-manifold $\tilde{W}$, we have

$$
\sigma(\widetilde{W})=4 \sigma(W)-\sum_{i=1}^{3} \operatorname{sign}\left(g_{i}, \widetilde{W}\right) .
$$

For $i=1,2,3, W^{(i)}=\tilde{W} /\left(g_{i}\right)$ has an action of $\left(\boldsymbol{Z}_{2} \oplus \boldsymbol{Z}_{2}\right) /\left(g_{i}\right) \cong \boldsymbol{Z}_{2}$, generated by $h_{i}$, say. Applying $\left(^{*}\right)$ again, we get 


$$
\sigma\left(W^{(i)}\right)=2 \sigma(W)-\operatorname{sign}\left(h_{i}, W^{(i)}\right),
$$

By the proof of the proposition on page 415 of [2]

$$
\operatorname{sign}\left(h_{i}, W^{(i)}\right)=\frac{1}{2} \sum_{j \neq i} \operatorname{sign}\left(g_{j}, \tilde{W}\right) .
$$

Hence

$$
\sum_{i=1}^{3} \operatorname{sign}\left(h_{i}, W^{(i)}\right)=\sum_{i=1}^{3} \operatorname{sign}\left(g_{i}, \widetilde{W}\right) .
$$

The result now follows from equations (1), (2) and (3).

We also need

LEMMA 2.

$$
\begin{aligned}
& \bar{e}\left(F_{1}^{(2)}\right)=2 \bar{e}\left(F_{1}\right), \quad \bar{e}\left(F_{2}^{(1)}\right)=2 \bar{e}\left(F_{2}\right), \\
& \bar{e}(F)=\bar{e}\left(F_{1}\right)+\bar{e}\left(F_{2}\right)-2 L k\left(\bar{l}_{1}, \bar{l}_{2}\right) .
\end{aligned}
$$

(Note that $L k\left(\bar{l}_{1}, \bar{l}_{2}\right)$ is well-defined, since $\bar{l}_{1}$ and $\bar{l}_{2}$ are both nullhomologous.)

Proof. To prove the first statement, let $V_{1}$ be an oriented surface in $M$ with $\partial V_{1}=-\bar{l}_{1}$. Let the inverse image of $V_{1}$ in $\partial W^{(2)}$ be $V_{1}^{(2)}$, a 2-fold branched cover (possibly disconnected) of $V_{1}$. Let $\bar{l}_{1}^{\prime}$ be the (oriented) boundary of a section of the normal 1-sphere bundle of $F_{1}$; its inverse image $\bar{l}_{1}^{(2)}$ in $\partial W^{(2)}$ is the boundary of a corresponding section for $F_{1}^{(2)}$. Then

$$
\bar{e}\left(F_{1}^{(2)}\right)=\bar{l}_{1}^{(2) \prime} \cdot V_{1}^{(2)}=2 \bar{l}_{1}^{\prime} \cdot V_{1}=2 \bar{e}\left(F_{1}\right) .
$$

Similarly, $\bar{e}\left(F_{2}^{(1)}\right)=2 \bar{e}\left(F_{2}\right)$. Finally, we may assume that $\bar{l}_{1}^{\prime}$ does not meet $l_{2}$, and is homologous to $\bar{l}_{1}$ in $M-l_{2}$. Extending in the obvious way the notation already introduced, we then have

$$
\begin{aligned}
\bar{e}(F) & =\left(\bar{l}_{1}^{\prime} \cup \bar{l}_{2}^{\prime}\right) \cdot\left(V_{1} \cup V_{2}\right) \\
& =\bar{l}_{1}^{\prime} \cdot V_{1}+\bar{l}_{2}^{\prime} \cdot V_{2}+\bar{l}_{1}^{\prime} \cdot V_{2}+\bar{l}_{2}^{\prime} \cdot V_{1} \\
& =\bar{e}\left(F_{1}\right)+\bar{e}\left(F_{2}\right)-2 L k\left(\bar{l}_{1}, \bar{l}_{2}\right) .
\end{aligned}
$$

5. From equations (i)-(iv), together with Lemmas 1 and 2 , one easily obtains

$$
\sigma\left(\bar{l}_{1}, p_{1}\right)+\sigma(\bar{l}, p)+\operatorname{Lk}\left(\bar{l}_{1}, \bar{l}_{2}\right)=\sigma\left(\bar{l}_{2}, p_{2}\right)+\sigma\left(\bar{l}_{1}^{(2)}, p_{1}^{(2)}\right) .
$$

Now suppose $M=S^{3}$ and $l_{2}$ is the unknot. Then $\partial W^{(2)}$ is also $S^{3}$, and $\sigma\left(\bar{l}_{2}, p_{2}\right)=0$, so the above equation becomes 


$$
\sigma\left(\bar{l}_{1}^{(2)}\right)=\sigma\left(\bar{l}_{1}\right)+\sigma(\bar{l})+\operatorname{Lk}\left(\bar{l}_{1}, \bar{l}_{2}\right) .
$$

If, further, $l_{1}$ has only one component, then

$$
\sigma(\bar{l})+\operatorname{Lk}\left(\bar{l}_{1}, \bar{l}_{2}\right)=\xi(l)
$$

so we obtain

$$
\sigma\left(\bar{l}_{1}^{(2)}\right)=\sigma\left(l_{1}\right)+\xi(l) .
$$

Theorem 1 is the special case in which $l_{1}^{(2)}$ has only one component.

REMARK. Using equations (i), (ii), (iii) and (vi) we obtain instead the relation

$$
\sigma(\phi, \widetilde{p})+\sigma(\bar{l}, p)+\operatorname{Lk}\left(\bar{l}_{1}, \bar{l}_{2}\right)=\sigma\left(\bar{l}_{1}, p_{1}\right)+\sigma\left(\bar{l}_{2}, p_{2}\right) .
$$

If $M=S^{3}$, this can be written as

$$
\sigma(\phi, \widetilde{p})+\xi(l)=\xi\left(l_{1}\right)+\xi\left(l_{2}\right) .
$$

\section{REFERENCET}

1. M. F. Atiyah and I. M. Singer, The index of elliptic operators III, Ann. of Math., (2), 87 (1968), 546-604.

2. F. Hirzebruch, Free involutions on manifolds and some elementary number theory, Symposia Mathematica (Instituto Nazionale di Alta Matematica, Roma) Vol. V. Academic Press (1971), 411-419.

3. L. Kauffman and L. Taylor, Signature of links, Trans. Amer. Math. Soc., 216 (1976), 351-365.

4. K. Murasugi, On the signature of links, Topology, 9 (1970), 283-298.

Received April 17, 1978.

The University of TeXas

AUstin, TX 78712 


\section{PACIFIC JOURNAL OF MATHEMATICS}

\section{EDITORS}

DoNALD BABBITT (Managing Editor)

University of California

Los Angeles, California 90024

HUGo Rossi

University of Utah

Salt Lake City, UT 84112

C. C. MOORE and ANDREW OGG

University of California

Berkeley, CA 94720
J. DUGUNDJI

Department of Mathematics University of Southern Californı Los Angeles, California 90007

R. Finn and J. Milgram Stanford University Stanford, California 94305

\section{ASSOCIATE EDITORS}

E. F. BECKENBACH

B. H. Neumann
F. WOLF

K. YOSHIDA

\section{SUPPORTING INSTITUTIONS}

UNIVERSITY OF BRITISH COLUMBIA CALIFORNIA INSTITUTE OF TECHNOLOGY UNIVERSITY OF CALIFORNIA MONTANA STATE UNIVERSITY UNIVERSITY OF NEVADA, RENO NEW MEXICO STATE UNIVERSITY OREGON STATE UNIVERSITY UNIVERSITY OF OREGON
UNIVERSITY OF SOUTHERN CALIFORNIA STANFORD UNIVERSITY UNIVERSITY OF HAWAII UNIVERSITY OF TOKYO UNIVERSITY OF UTAH WASHINGTON STATE UNIVERSITY UNIVERSITY OF WASHINGTON 


\section{Pacific Journal of Mathematics}

Vol. 82, No. $1 \quad$ January, 1979

Werner Bäni, Subspaces of positive definite inner product spaces of countable dimension ...................................... 1

Marilyn Breen, The dimension of the kernel of a planar set..............

Kenneth Alfred Byrd, Right self-injective rings whose essential right ideals

are two-sided

Patrick Cousot and Radhia Cousot, Constructive versions of Tarski's fixed

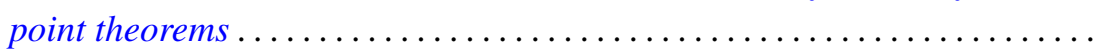

Ralph S. Freese, William A. Lampe and Walter Fuller Taylor, Congruence lattices of algebras of fixed similarity type. $I \ldots \ldots \ldots \ldots \ldots \ldots \ldots$

Cameron Gordon and Richard A. Litherland, On a theorem of Murasugi .....

Mauricio A. Gutiérrez, Concordance and homotopy. I. Fundamental

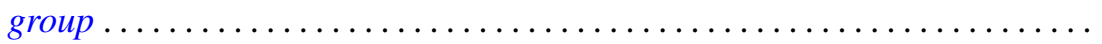

Richard I. Hartley, Metabelian representations of knot groups .............

Ted Hurley, Intersections of terms of polycentral series of free groups and free

Lie algebras ........................................

Roy Andrew Johnson, Some relationships between measures ............ 117

Oldřich Kowalski, On unitary automorphisms of solvable Lie algebras .......

Kee Yuen Lam, $K O$-equivalences and existence of nonsingular bilinear

maps...................................................

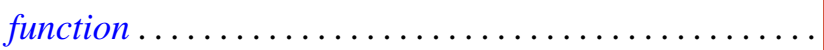

Robert A. Messer and Alden H. Wright, Embedding open 3-manifolds in compact 3-manifolds ............................

Gerald Ira Myerson, A combinatorial problem in finite fields. I . .

James Nelson, Jr. and Mohan S. Putcha, Word equations in a band of paths.

Baburao Govindrao Pachpatte and S. M. Singare, Discrete generalized Gronwall inequalities in three independent variables . .

William Lindall Paschke and Norberto Salinas, $C^{*}$-algebras associated with free products of groups ........................

Bruce Reznick, Banach spaces with polynomial norms ....

David Rusin, What is the probability that two elements of a finite group

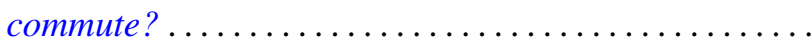

M. Shafii-Mousavi and Zbigniew Zielezny, On hypoelliptic differential operators of constant strength ...

Joseph Gail Stampfli, On selfadjoint derivation ranges .... . . .

Robert Charles Thompson, The case of equality in the matrix-valued triangle

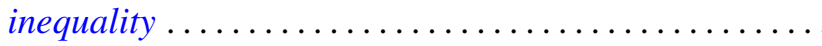

Marie Angela Vitulli, The obstruction of the formal moduli space in the negatively graded case. 